\title{
Adaptive Admission Control with Bandwidth Quantization and Dynamic Reallocation for Mobile WiMAX
}

\author{
Kai-Wei Ke, Ho-Ting Wu, D. Jayasakthi, and Shiao-Ting Huang
}

\begin{abstract}
The paper utilized the idea of bandwidth quantization and proposed a dynamic and adaptive bandwidth reallocation for admission control in mobile WiMAX networks. Based upon four QoS mechanisms (excluding the best effort), adaptively degrade and recover bandwidth to an existing user may improve the system performance but with tradeoff of complexity. Hand over and new calls were taken into account. The paper did analytical evaluations with multidimensional Markov chain; bandwidth utilization and blocking/dropping probability were the performance indices for comparison. The results presented that the utilization was increased and those probabilities were reduced simultaneously.
\end{abstract}

Index Terms-QoS, adaptive admission control, bandwidth quantization and reallocation, hand over

\section{INTRODUCTION}

The IEEE802.16 standard has been developed by IEEE802.16 Working Group since 1999. In June2004, a revision of IEEE Std 802.16-2001, called IEEE Std 802.16-2004, was approved [1]. In December 2005, the amendment project IEEE $802.16 \mathrm{e}$ was approved. This amendment expands the IEEE 802.16 fixed access system into a combined fixed and mobile system, allowing a single base station (BS) to support both fixed and mobile terminals. This amendment was later included in the newest revision of the standard, called IEEE Std 802.16-2009, in May 2009 [2].

There were many researches discussed the QoS architecture (since it is not specified clearly by IEEE 802.16 series specifications) and resource allocation for WiMAX networks, some of the papers designed admission control mechanism by considering delay constrain [3]-[10]. In view of related studies, those methods proposed by references [8]-[10] were similar to our work. They applied the concept of bandwidth degradation in the design of admission control and modeled the strategy by continuous-time Markov chain to analyze the performance. However, there were two common drawbacks presented in the papers: (1) Once the bandwidth degradation was activated to admit a new connection in, it was imposed on all existing connections in

Manuscript received January 15, 2013; revised April 2, 2013. This work was supported in part by the National Science Council, Taiwan, ROC, under Grant NSC100-2221-E -027-076.

Kai-Wei and Ho-Ting $\mathrm{Wu}$ are with the Department of CSIE, National Taipei University of Technology (NTUT), Taiwan, ROC (e-mail: kwk@csie.ntut.edu.tw, htwu@csie.ntut.edu.tw).

D. Jayasakthi is with CSIE institute, NTUT (e-mail: sakthi.venmathi@gmail.com).

Shiao-Ting Huang is with NTUT, and now she is with Qisda Co. Ltd., Taiwan, ROC. progress, this may yield excess bandwidth and cause bandwidth waste as well as performance degradation due to unnecessary reduction of the granted bandwidth of some connections, (2) The excess bandwidth taken back from existing connections is no longer reused while a new connection is granted, this causes the bandwidth waste even worse.

The idea of bandwidth quantization was first proposed by C. T. Lea et al [11]-[12] for broadband networks. In those work, it was shown that supporting continuous data rate (any value as requested) does not guarantee a good performance from the viewpoint of resource utilization/throughput and blocking probability, and proved that supporting only certain fixed data rates (called quantized bandwidth) would be appropriate in terms of system performance, but reduce the complexity drastically.

This paper is based upon the quantization concept and design a adaptive Call Admission Control (CAC) strategy that dynamically reallocate bandwidth by taking back from existing connections (i.e. bandwidth degrade) and reassigned excess bandwidth to incoming connections (bandwidth upgrade), and is abbreviated as BDR_CAC. In addition to resolving the issues in [3]-[5], effectively increasing radio resource utilization and decreasing blocking and dropping probabilities for IEEE 802.16e/Mobile WiMAX networks with QoS support, are the major concerns of the study.

The rest of the paper is organized as follows. Section II describes the proposed admission control, Section III builds the analytical model for analysis, Section IV illustrates the numerical results, and finally Section $\mathrm{V}$ draws the conclusion.

\section{ADAPTIVE ADMISSION CONTROL WITH BANDWIDTH QUANTIZATION AND DYNAMIC REALLOCATION}

Consider a Mobile WiMAX system supporting finite amount of fixed data rates and it is deployed similar to the public cellular phone system, as shown in Fig. 1, where each base station handles both new and hand over connection requests and a hand over request is given higher priority.

Without loss of generality, four types of eight different bandwidth requirements, UGS, ertPS, rtPS1, rtPS2, rtPS3, nrtPS1, nrtPS2, nrtPS3, are discussed in this paper. The best effort (BE) is not specifically considered in admission control due to its QoS-free property. The precedence for four service types is UGS $>$ ertPS $>$ rtPS $>$ nrtPS, and thus the following priority for handling various connection requests can be established: H-UGS $>$ H-ertPS $>$ N-UGS $>$ N-ertPS $>$ H-rtPS $>\mathrm{N}$-rtPS $>\mathrm{H}$-nrtPS $>\mathrm{N}$-nrtPS in which $\mathrm{N}$ stands for a new and $\mathrm{H}$ for a hand over request, respectively. Also, assume 
three bandwidth requirements for rtPS services are $\operatorname{rtPS} 1>$ rtPS2 $>$ rtPS3 and for nrtPS are nrtPS1 $>$ nrtPS2 $>$ nrtPS3. That is, $r$ PS $1 \leq$ bandwidth request for $\mathrm{rtPS}$ service $\leq \mathrm{rtPS} 3$, and nrtPS $1 \leq$ bandwidth request for nrtPS $\leq$ nrtPS3.

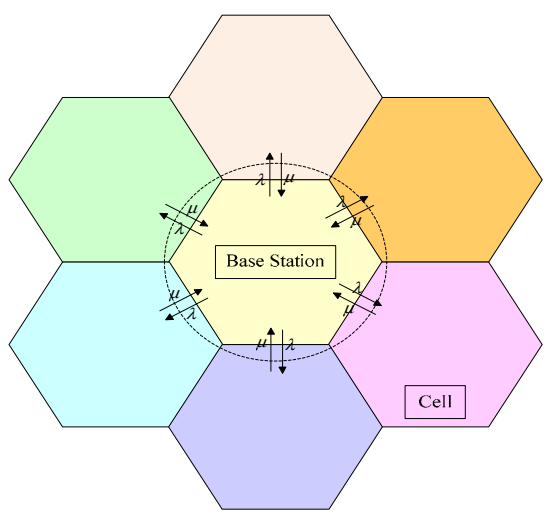

Fig. 1. Deployment of a WiMAX network.

Fig. 2 shows the architecture of BDR_CAC. As the flow shows, different service type of connection will be processed by its corresponding CAC mechanism (e.g. UGS connection block, Handoff rtPS connection block, etc.). Furthermore, as a connection leaves the BS, either terminated normally or hand over to an adjacent BS, the Recovery Algorithm will be activated for bandwidth upgrade by adding up bandwidth to some existing connections as they were taken by the BS (borrowed) to accommodate a new request in the first place.

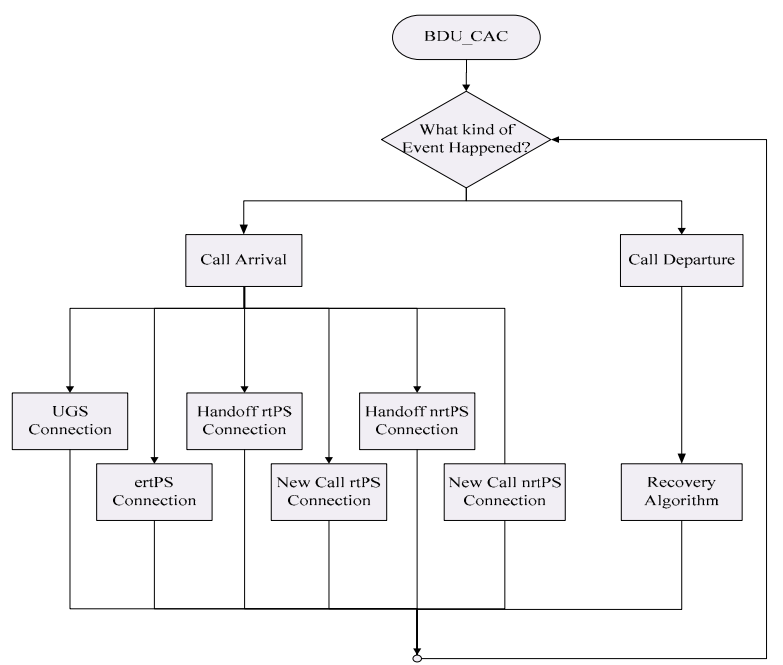

Fig. 2. The architecture of BDU_CAC.

Let the 8-tuple vector $(i, j, k, l, m, n, p, q)$ be the system state indicating that there are $i$ 's UGS, $j$ 's ertPS $1, k$ 's rtPS1, $l$ 's rtPS2, $m$ 's rtPS3, $n$ 's nrtPS1, $p$ 's nrtPS2, and $q$ 's nrtPS3 connections in progress (i.e. handled by a BS currently), and $b_{i}, 1 \leq i \leq 8$ be the corresponding bandwidth requirements for above eight connection types. Because of limitations of space, we illustrate detailed flow of CAC blocks in Fig. 2 for an ertPS connection request (Fig. 3) and bandwidth recovery (update) algorithm when a connection leaves the system (Fig. 4). The symbols are interpreted below: R $\left(\right.$ or $\left.B_{\text {rem }}\right)=$ residual capacity, $D_{r}=$ the number of $\operatorname{rtPS}$ connections that can be chosen for upgrading their bandwidth $=\left\lfloor b i+R / \Delta_{r}\right\rfloor$, while $D_{n}$

$=$ the number of nrtPS connections that can be chosen for upgrading their bandwidth $=\left\lfloor B+R-\left(B_{\text {used }}+B_{\text {rem }}\right) \cdot \Delta_{r} / \Delta_{n}\right\rfloor^{+}$. The rule for bandwidth recovery is: upgrading rtPS connections first and then nrtPS if there is residue bandwidth available; for both rtPS and nrtPS connections to be upgraded, the priority gives to $\operatorname{rtPS} 3>\operatorname{rtPS} 2>\operatorname{nrtPS} 3>\operatorname{nrtPS} 2$. The details of upgrading procedure is shown in Fig. 4.

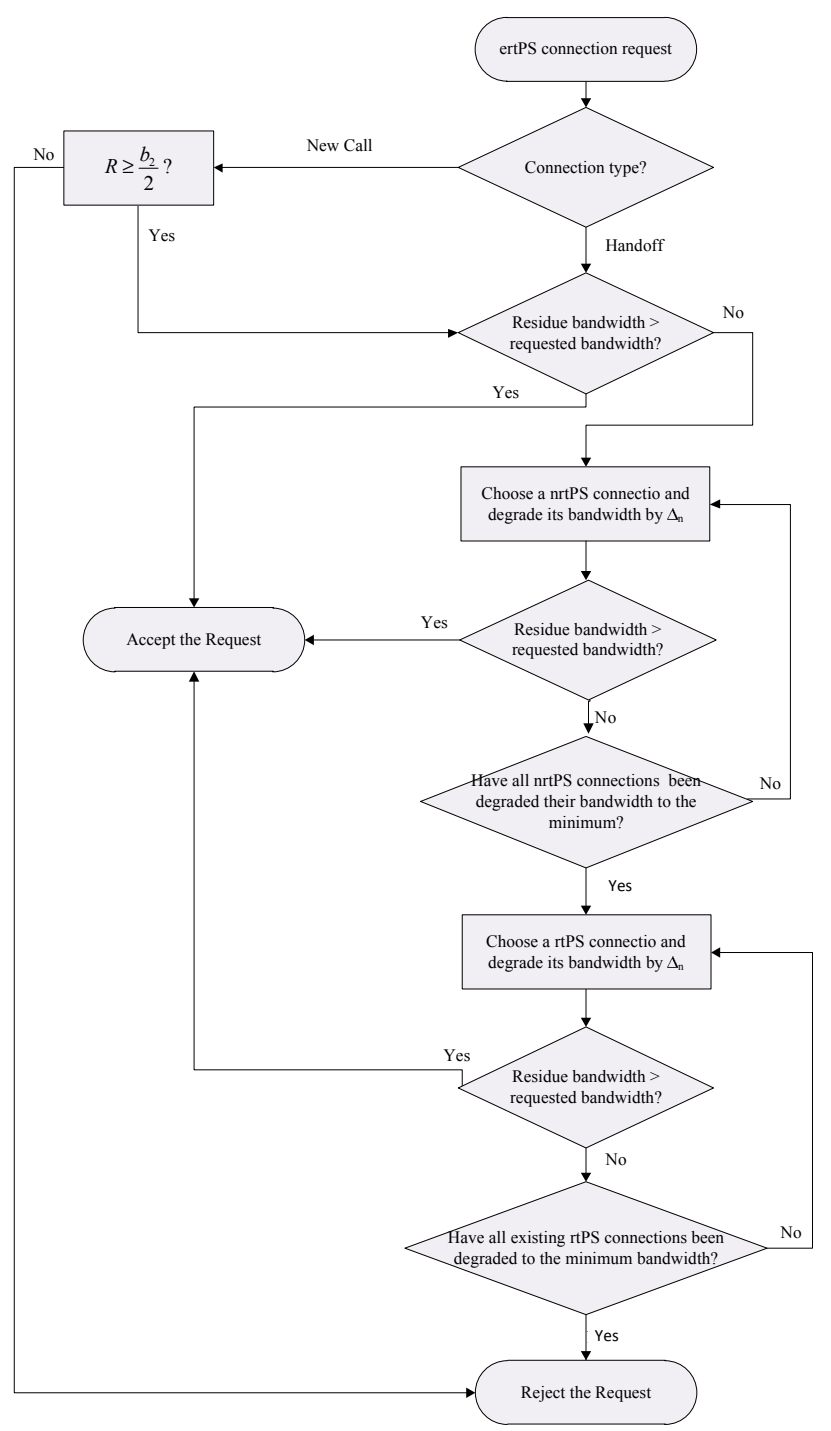

Fig. 3. CAC for ertPS connection requests.

\section{System Model AND PERFORMANCE ANALYSIS}

A connection request is similar to a call request in PSTN or cellular system, and the Poisson arrival process is assumed in analysis. Let $\left[\lambda_{n u}, \lambda_{n e}, \lambda_{n r}\right.$, and $\left.\lambda_{n n}\right]$ and $\left[\lambda_{h u}, \lambda_{h e}, \lambda_{h r}, \lambda_{h n}\right]$ be the arrival rates for new and hand over UGS, ertPS, rtPS, and nrtPS connection requests, respectively. Thus, to a base station, the total arrival rate will be $\lambda_{T}=\lambda_{h u}+\lambda_{h e}+\lambda_{h r}+\lambda_{h n}+\lambda_{n u}+\lambda_{n e}+\lambda_{n r}+\lambda_{n n}$. The dwelling time inside the cell of a BS and holding time of different service types are independently and exponential distributed. Their mean values are represented by $1 / \mu_{u}, 1 / \mu_{e}, 1 / \mu_{r l}, 1 / \mu_{r 2}$, $1 / \mu_{r 3}, 1 / \mu_{n 1}, 1 / \mu_{n 2}$, and $1 / \mu_{n 3}$. The granted bandwidth will be occupied also in exponential distribution [10] with mean value of $\mu_{T}=1 /\left(\mu_{u}+\mu_{e}+\mu_{r 1}+\mu_{r 2}+\mu_{r 3}+\mu_{n 1}+\mu_{n 2}+\mu_{n 3}\right)$, where the subscripts [u, e, r, n] represents [UGS, ertPS, rtPS, nrtPS (with different rates)] connections, for both new and hand 
over ones, respectively.

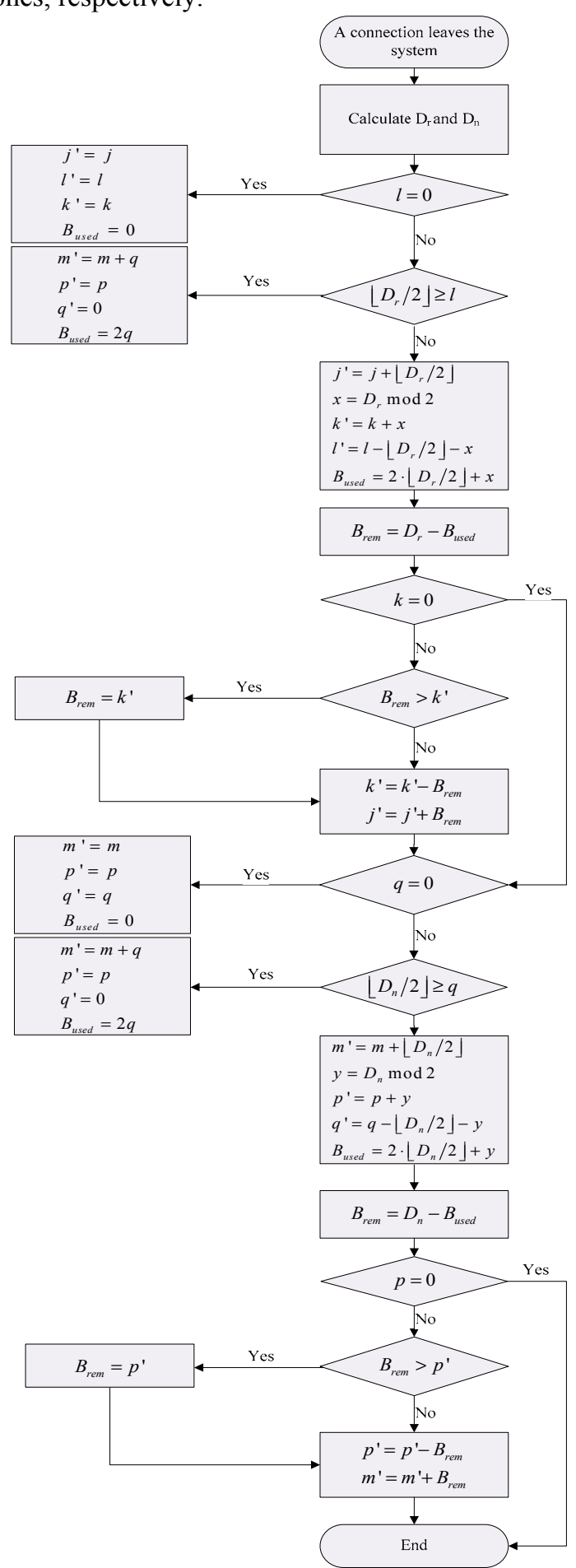

Fig. 4. Bandwidth recovery algorithm (applying bandwidth upgrade).

The system operation can be modeled by an eight-dimension Markov chain with the same state vector $(i, j$, $k, l, m, n, p, q)$ as defined in section 2. The bandwidth requirement can be described by an 8-tuple vector of $\left(b_{1}, b_{2}, b_{3}, b_{4}, b_{5}, b_{6}, b_{7}, b_{8}\right)$. In addition, define $\Delta_{r}=b_{3}-b_{4}$ $=b_{4}-b_{5}$ as the bandwidth deviation for three rtPS connection types, it represents the amount of bandwidth can be degraded (released) or upgraded (recovered) by one step; Similarly, $\Delta_{n}$ $=b_{6}-b_{7}=b_{7}-b_{8}$ will be those for three nrtPS connection types, and thus the bandwidth requirement for connections $\left(b_{1}, b_{2}, b_{3}, b_{4}, b_{5}, b_{6}, b_{7}, b_{8}\right)$ equals to $\left(b_{1}, b_{2}, 3 \Delta_{r}, 2 \Delta_{r}, \Delta_{r}, 3 \Delta\right.$ $\left.{ }_{n}, 2 \Delta_{n}, \Delta_{n}\right)$.
All allowable states form the state space $\mathrm{S}$ :

$$
\begin{aligned}
& S=\left\{(i, j, k, l, m, n, p, q) \mid i \cdot b_{1}+j \cdot b_{2}+k \cdot b_{3}+l \cdot b_{4}\right. \\
& \left.+m \cdot b_{5}+n \cdot b_{6}+p \cdot b_{7}+q \cdot b_{8} \leq C\right\}
\end{aligned}
$$

where $C$ is the total amount of resource. Define $\pi_{(i, j, k, l, m, n, p, q)}$ as the steady-state probability for state $(i, j, k, l, m, n, p, q)$ and $\pi_{(i, j, k, l, m, n, p, q)}=0$, if $(i, j, k, l, m, n, p, q) \notin S$. For all legal states $(i, j, k, l, m, n, p, q) \in S$,

$$
\sum_{(i, j, k, l, m, n, p, q) \in S} \pi_{(i, j, k, l, m, n, p, q)}=1
$$

For a general system state $(i, j, k, l, m, n, p, q)$, all possible transitions can be described in Fig. 5, with indicating functions defined by:

$$
\begin{aligned}
& \delta_{u}=\left\{\begin{array}{lr}
1, & \text { if }\left\{R+\left[(2 k+l) \cdot \Delta_{r}+(2 n+p) \cdot \Delta_{n}\right]\right\} \geq b_{1}, \text { and }\left(i+1, j, k^{\prime}, l^{\prime}, m^{\prime}, n^{\prime}, p^{\prime}, q^{\prime}\right) \in S \\
0, & \text { otherwise }
\end{array}\right. \\
& \delta_{u}^{+}= \begin{cases}1, & \text { if }\left(i, j, k^{\prime}, l^{\prime}, m^{\prime}, n^{\prime} p^{\prime}, q^{\prime}\right)=(i, j, k, l, m, n, p, q), \text { and }\left(i+1, j, k^{\prime}, l^{\prime}, m^{\prime}, n^{\prime}, p^{\prime}, q^{\prime}\right) \in S \\
0, & \text { otherwise }\end{cases} \\
& \delta_{u}^{-}= \begin{cases}1, & \text { if }\left(i, j, k^{\prime}, l^{\prime}, m^{\prime}, n^{\prime} p^{\prime}, q^{\prime}\right)=(i, j, k, l, m, n, p, q), \text { and }\left(i-1, j, k^{\prime}, l^{\prime}, m^{\prime}, n^{\prime}, p^{\prime}, q^{\prime}\right) \in S \\
0, & \text { otherwise }\end{cases} \\
& \delta_{e}=\left\{\begin{array}{lr}
1, & \text { if }\left\{R+\left[(2 k+l) \cdot \Delta_{r}+(2 n+p) \cdot \Delta_{n}\right]\right\} \geq b_{2}, \text { and }\left(i, j+1, k^{\prime}, l^{\prime}, m^{\prime}, n^{\prime}, p^{\prime}, q^{\prime}\right) \in S \\
0, & \text { otherwise }
\end{array}\right. \\
& \delta_{e}^{+}=\left\{\begin{array}{lc}
1, & \text { if }\left(i, j, k^{\prime}, l^{\prime}, m^{\prime}, n^{\prime} p^{\prime}, q^{\prime}\right)=(i, j, k, l, m, n, p, q), \text { and }\left(i, j+1, k^{\prime}, l^{\prime}, m^{\prime}, n^{\prime}, p^{\prime}, q^{\prime}\right) \in S \\
0, & \text { otherwise }
\end{array}\right. \\
& \delta_{e}^{-}= \begin{cases}1, & \text { if }\left(i, j, k^{\prime}, l^{\prime}, m^{\prime}, n^{\prime} p^{\prime}, q^{\prime}\right)=(i, j, k, l, m, n, p, q), \text { and }\left(i, j-1, k^{\prime}, l^{\prime}, m^{\prime}, n^{\prime}, p^{\prime}, q^{\prime}\right) \in S \\
0, & \text { otherwise }\end{cases} \\
& \delta_{h r}=\left\{\begin{array}{lr}
1, & \text { if }\left\{R+\left[(2 j+k) \cdot \Delta_{r}+(2 m+p) \cdot \Delta_{n}\right]\right\} \geq b_{5}, \text { and }\left(i, j, k^{\prime}, l^{\prime}, m^{\prime}, n^{\prime} p^{\prime}, q^{\prime}\right) \in S \\
0, & \text { otherwise }
\end{array}\right. \\
& \delta=\left\{\begin{array}{lc}
1, & \text { if }\left(i, j, k^{\prime}, l^{\prime}, m^{\prime}, n^{\prime}, p^{\prime}, q^{\prime}\right)=(i, j, k, l, m, n, p, q), \text { and }\left(i, j, k^{\prime}, l^{\prime}, m^{\prime}, n^{\prime}, p^{\prime}, q^{\prime}\right) \in S \\
0, & \text { otherwise }
\end{array}\right. \\
& \delta_{n r}=\left\{\begin{array}{cc}
1, & \text { if } R+\left[(2 n+p) \cdot \Delta_{n}\right] \geq b_{5}, \text { and }\left(i, j, k^{\prime}, l^{\prime}, m^{\prime}, n^{\prime}, p^{\prime}, q^{\prime}\right) \in S \\
0, & \text { otherwise }
\end{array}\right. \\
& \delta_{h n}=\left\{\begin{array}{cc}
1, & \text { if }\left[R+(2 n+p) \cdot \Delta_{n}\right] \geq b_{8}, \text { and }\left(i, j, k, l, m, n^{\prime} p^{\prime}, q^{\prime}\right) \in S \\
0, & \text { otherwise }
\end{array}\right. \\
& \delta_{n}=\left\{\begin{array}{lc}
1, & i f\left(i, j, k, l, m, n^{\prime}, p^{\prime}, q^{\prime}\right)=(i, j, k, l, m, n, p, q), \text { and }\left(i, j, k, l, m, n^{\prime}, p^{\prime}, q^{\prime}\right) \in S \\
0, & \text { otherwise }
\end{array}\right. \\
& \delta_{n n 1}=\left\{\begin{array}{cc}
1, & \text { if } R \geq b_{6}, \text { and }(i, j, k, l, m, n+1, p, q) \in S \\
0, & \text { otherwise }
\end{array}\right. \\
& \delta_{n n 2}=\left\{\begin{array}{cc}
1, & \text { if } R \geq b_{7}, \text { and }(i, j, k, l, m, n, p+1, q) \in S \\
0, & \text { otherwise }
\end{array}\right.
\end{aligned}
$$$$
\delta_{n n 3}=\left\{\begin{array}{cc}
1, & \text { if } R \geq b_{8}, \text { and }(i, j, k, l, m, n, p, q+1) \in S \\
0, & \text { otherwise }
\end{array}\right.
$$

Based upon the state diagram, the balance equations can be derived in (4), with

$$
\lambda_{u t}=\left\{\begin{array}{cc}
\lambda_{n u}+\lambda_{n u}, & \text { if } R \geq \frac{b_{1}}{2} \\
\lambda_{n u}, & \text { otherwise }
\end{array} \text { and } \lambda_{t e}=\left\{\begin{array}{cc}
\lambda_{h e}+\lambda_{n e}, & \text { if } R \geq \frac{b_{2}}{2} \\
\lambda_{n e}, & \text { otherwise }
\end{array}\right.\right.
$$

And the steady-state probabilities can then be calculated.

Finally, the performance indices, the blocking probability for new connection requests $P_{C B P}$, the dropping probability for hand over connection requests, and utilization of radio resource can then be calculated as follows. 


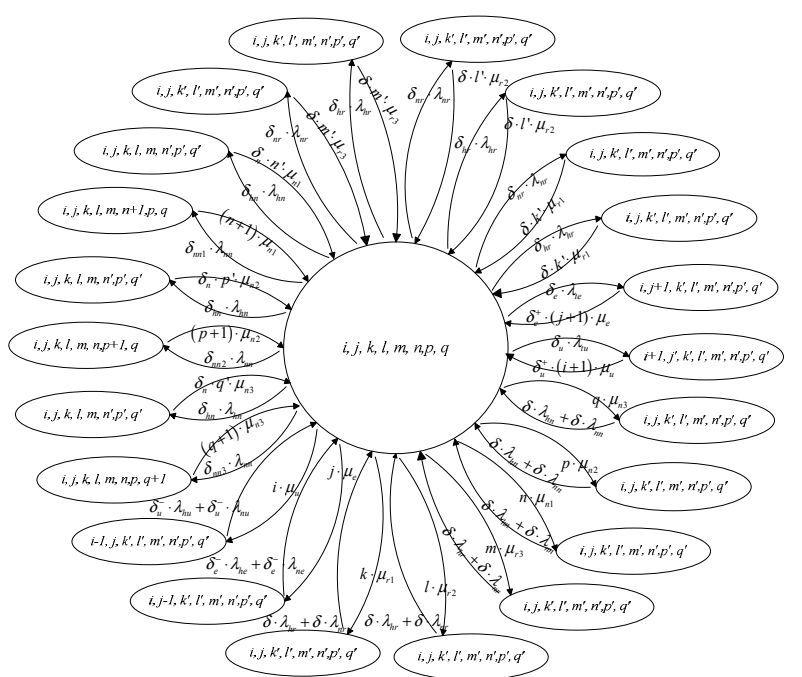

Fig. 5. State transitions for a state $(i, j, k, l, m, n, p, q)$ in the BDU_CAC.

$\pi_{(i, j, k, l, m, p, q)} \cdot\left\{\delta_{u} \cdot \lambda_{t u}+\delta_{e} \cdot \lambda_{t e}+\delta_{h r} \cdot \lambda_{h r}+\delta_{n r} \cdot \lambda_{n r}+\delta_{h r} \cdot \lambda_{h r}+\delta_{n r} \cdot \lambda_{n r}+\delta_{h r} \cdot \lambda_{h r}+\delta_{n r} \cdot \lambda_{n r}\right.$ $+\delta_{h n} \cdot \lambda_{n n}++\delta_{h n} \cdot \lambda_{h n}+\delta_{h n} \cdot \lambda_{h n}+\lambda_{n n} \cdot\left[\delta_{n n 1}+\delta_{n n 2}+\delta_{n n 3}\right]+i \cdot \mu_{u}+j \cdot \mu_{r 1}+k \cdot \mu_{r 2}$ $\left.\left.+l \cdot \mu_{r 3}+m \cdot \mu_{n 1}+p \cdot \mu_{n 2}+q \cdot \mu_{n 3}\right]\right\}$

$=\delta_{u}^{+} \cdot(i+1) \cdot \mu_{u} \cdot \pi_{\left(i+1, j^{\prime}, k^{\prime}, l^{\prime}, m^{\prime}, n^{\prime}, p^{\prime}, q^{\prime}\right)}+\delta_{e}^{+} \cdot(j+1) \cdot \mu_{e} \cdot \pi_{\left(i, j+1, k^{\prime}, l^{\prime}, m^{\prime}, n^{\prime}, p^{\prime}, q^{\prime}\right)}$ $+\mu_{r 1} \cdot\left[\delta \cdot k^{\prime} \cdot \pi_{\left(i, j, k^{\prime}, l^{\prime}, m^{\prime}, n^{\prime}, p^{\prime}, q^{\prime}\right)}+\delta \cdot k^{\prime} \cdot \pi_{\left(i, j, k^{\prime}, l^{\prime}, m^{\prime}, n^{\prime}, p^{\prime}, q^{\prime}\right)}\right]+\mu_{r 2} \cdot\left[\delta \cdot l^{\prime} \cdot \pi_{\left(i, j, k^{\prime}, l^{\prime}, m^{\prime}, n^{\prime}, p^{\prime}, q^{\prime}\right)}\right.$ $\left.+\delta \cdot l^{\prime} \cdot \pi_{\left(i, j, k^{\prime}, l^{\prime}, m^{\prime}, n^{\prime}, p^{\prime}, q^{\prime}\right)}\right]+\mu_{r 3} \cdot\left[\delta \cdot m^{\prime} \cdot \pi_{\left(i, j, k^{\prime}, l^{\prime}, m^{\prime}, n^{\prime}, p^{\prime}, q^{\prime}\right)}+\delta \cdot m^{\prime} \cdot \pi_{\left(i, j, k^{\prime}, l^{\prime}, m^{\prime}, n^{\prime}, p^{\prime}, q^{\prime}\right)}\right]$ $+\mu_{n 1} \cdot\left[\delta_{n} \cdot n^{\prime} \cdot \pi_{\left(i, j, k, l, m, n, n^{\prime}, q^{\prime} q^{\prime}\right)}+(n+1) \cdot \pi_{(i, j, k, l, m, n+1, p, q)}\right]+\mu_{n 2} \cdot\left[\delta_{n} \cdot p^{\prime} \cdot \pi_{\left(i, j, k, k, m, n^{\prime}, p^{\prime}, q^{\prime}\right)}\right.$ $\left.+(p+1) \cdot \pi_{(i, j, k, l, m, n, p+1, q)}\right]+\mu_{n 3} \cdot\left[\delta_{n} \cdot q^{\prime} \cdot \pi_{\left(i, j, k, l, l, n, n^{\prime}, p^{\prime}, q^{\prime}\right)}+(q+1) \cdot \pi_{(i, j, k, l, m, n, p, q+1)}\right]$ $+\left(\delta_{u}^{-} \cdot \lambda_{h u}+\delta_{u}^{-} \cdot \lambda_{n u}\right) \cdot \pi_{\left(i-1, j, k^{\prime}, l^{\prime}, m^{\prime}, n^{\prime}, p^{\prime}, q^{\prime}\right)}+\left(\delta_{e}^{-} \cdot \lambda_{h e}+\delta_{e}^{-} \cdot \lambda_{n e}\right) \cdot \pi_{\left(i, j-1, k^{\prime}, l^{\prime}, m^{\prime}, n^{\prime}, p^{\prime}, q^{\prime}\right)}$ $+\left(\delta \cdot \lambda_{h r}+\delta \cdot \lambda_{n r}\right) \cdot \pi_{\left(i, j, k^{\prime}, l^{\prime}, m^{\prime}, n^{\prime}, p^{\prime}, q^{\prime}\right)}+\left(\delta \cdot \lambda_{h r}+\delta \cdot \lambda_{n r}\right) \cdot \pi_{\left(i, j, k^{\prime}, l^{\prime}, m^{\prime}, n^{\prime}, p^{\prime}, q^{\prime}\right)}$ $+\left(\delta \cdot \lambda_{h r}+\delta \cdot \lambda_{n r}\right) \cdot \pi_{\left(i, j, k^{\prime}, l^{\prime}, m^{\prime}, n^{\prime}, p^{\prime}, q^{\prime}\right)}+\left(\delta \cdot \lambda_{h n}+\delta \cdot \lambda_{n n}\right) \cdot \pi_{\left(i, j, k^{\prime}, l^{\prime}, m^{\prime}, n^{\prime}, p^{\prime}, q^{\prime}\right)}$ $+\left(\delta \cdot \lambda_{h n}+\delta \cdot \lambda_{n n}\right) \cdot \pi_{\left(i, j, k^{\prime}, l^{\prime}, m^{\prime}, n^{\prime}, p^{\prime}, q^{\prime}\right)}+\left(\delta \cdot \lambda_{h n}+\delta \cdot \lambda_{n n}\right) \cdot \pi_{\left(i, j, k^{\prime}, l^{\prime}, m^{\prime}, n^{\prime}, p^{\prime}, q^{\prime}\right)}$,

A. Blocking Probabilities for New Connections:

$B P_{U G S}=\sum_{s \in S_{B P} U G S} \pi_{s}$, where $S_{B P_{-} U G S}=\{s=(i, j, k, l, m, n, p, q)$ $\left.\left(R<\frac{b_{1}}{2}\right) \vee\left[(2 k+l) \Delta_{r}+(2 n+p) \Delta_{n}<\left(b_{1}-R\right)\right]\right\}$. $B P_{\text {ertPS }}=\sum_{s \in S_{B P \_ \text {ertPS }}} \pi_{s}$, where $S_{B P_{-} e r t P S}=\{s=(i, j, k, l, m, n, p, q) \mid$ $\left.\left(R<\frac{b_{2}}{2}\right) \vee\left[(2 k+l) \Delta_{r}+(2 n+p) \Delta_{n}<\left(b_{2}-R\right)\right]\right\}$.

$B P_{r t P S}=\sum_{s \in S_{B P_{-} r t P S}} \pi_{s}$, where $S_{B P_{-} r t P S}=\{s=(i, j, k, l, m, n, p, q) \mid$ $\left.\left[(2 n+p) \Delta_{n}<\left(b_{5}-R\right)\right]\right\}$.

$B P_{n r t P S}=\sum_{s \in S_{B P_{-} n t P S}} \pi_{S}$, where $S_{B P_{-} n r t P S}=\{s=(i, j, k, l, m, n, p, q) \mid$

$\left.\left(R<b_{6}\right) \vee\left(R<b_{7}\right) \vee\left(R<b_{8}\right)\right\}$.

\section{B. Dropping Probabilities for New Connections:}

$D P_{U G S}=\sum_{s \in S_{D P} U G S} \pi_{s}$, where $S_{D P_{-} U G S}=\{s=(i, j, k, l, m, n, p, q) \mid$

$\left.\left[(2 k+l) \Delta_{r}+(2 n+p) \Delta_{n}<\left(b_{1}-R\right)\right]\right\}$.

$$
\begin{aligned}
& D P_{\text {ertPS }}=\sum_{s \in S_{D P_{-} \text {ertPS }}} \pi_{s}, \text { where } S_{D P_{-} \text {ertPS }}=\{s=(i, j, k, l, m, n, p, q) \\
& \left.\left[(2 k+l) \Delta_{r}+(2 n+p) \Delta_{n}<\left(b_{2}-R\right)\right]\right\} . \\
& D P_{r t P S}=\sum_{s \in S_{D P_{-} r P S}} \pi_{s}, \text { where } S_{D P_{-} r t P S}=\{s=(i, j, k, l, m, n, p, q) \\
& \left.\left[(2 k+l) \Delta_{r}+(2 n+p) \Delta_{n}<\left(b_{5}-R\right)\right]\right\} . \\
& D P_{n r t P S}=\sum_{s \in S_{D P_{-} r t P S}} \pi_{s}, \text { where } S_{D P_{-} n r t P S}=\{s=(i, j, k, l, m, n, p, q) \\
& \left.(2 n+p) \Delta_{n}<\left(b_{8}-R\right)\right\} .
\end{aligned}
$$$$
\text { C. Resource Utilization: }
$$$$
U=\frac{\sum_{s \in S} \pi_{s} \cdot C_{s}}{C}, s \in S, C_{s}=i \cdot b_{1}+j \cdot b_{2}+\cdots+q \cdot b_{8} .
$$

\section{NUMERICAL RESUlTS AND Discussion}

In numerical evaluations, we assume that a frame duration $=5 \mathrm{~ms}$ with data rate of $350 \mathrm{~Kb} / \mathrm{s}$, and the total data rate for an uplink (assuming one-half of the frame) channel = $2.5 \mathrm{~ms} /$ frame $\times(350 \mathrm{~Kb} / \mathrm{s}) / 2 \times 200$ frame $/ \mathrm{s}=35 \mathrm{Mbps}$. Data rates for various connections of different service types, where the best effort (BE) is not considered. Also, assume that arrival rates $\lambda_{h u}=\lambda_{h e}=\lambda_{h r}=\lambda_{h n}=\lambda_{n u}=\lambda_{n e}=\lambda_{n r}=\lambda_{n n}$ and mean service times $\mu_{u}=\mu_{e}=\mu_{r 1}=\mu_{r 2}=\mu_{r 3}=\mu_{n 1}=\mu_{n 2}=\mu_{n 3}=0.2$.

TABLE I. QOS PARAMETERS USED IN NUMERICAL ANALYSIS.

\begin{tabular}{|c|c|c|c|}
\hline Service & Rate(Kbits) & Service & Rate(K bits) \\
\hline UGS & 32 & rtPS3 & 32 \\
\hline ertPS & 64 & nrtPS1 & 48 \\
\hline rtPS1 & 96 & nrtPS2 & 32 \\
\hline rtPS2 & 64 & nrtPS3 & 16 \\
\hline
\end{tabular}

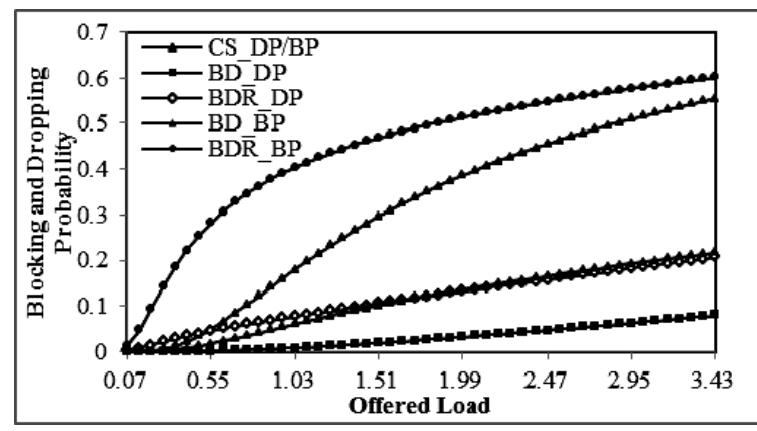

Fig. 6. Blocking and dropping probabilities for UGS connections.

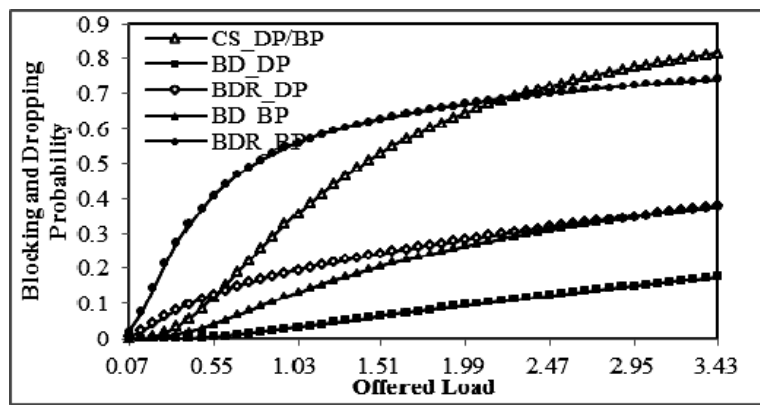

Fig. 7. Blocking and dropping probabilities for ertPS connections. 


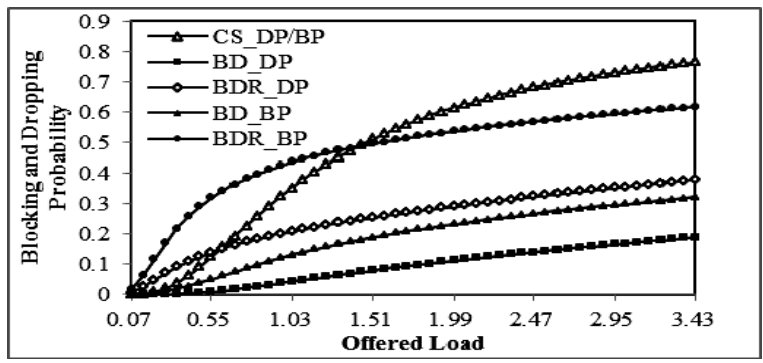

Fig. 8. Blocking and dropping probabilities for rtPS connections.

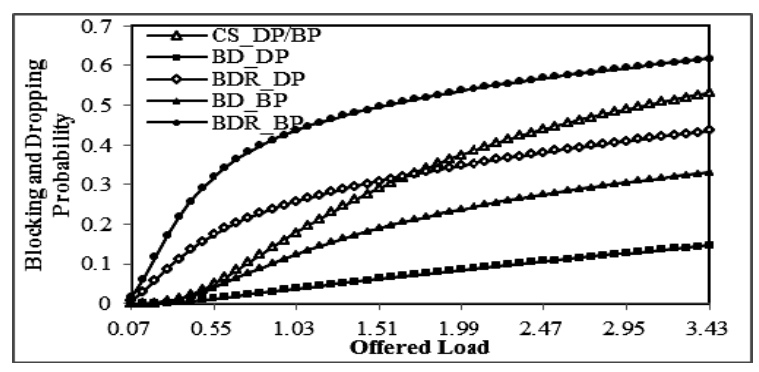

Fig. 9. Blocking and dropping probabilities for nrtPS connections.

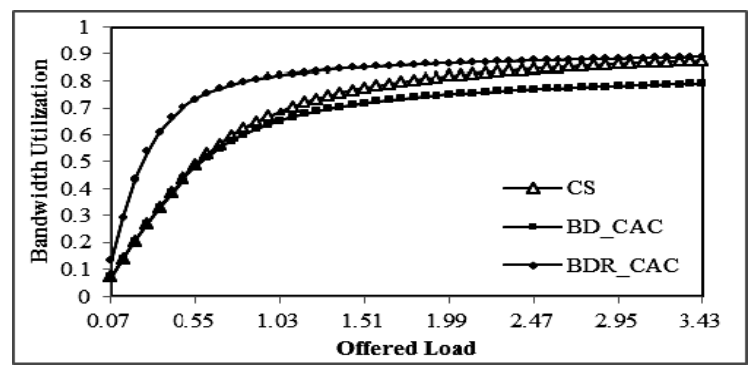

Fig. 10. Comparison of bandwidth utilization.

The performance of BDR_CAC are compared to complete-sharing control (CS) and Bandwidth Degrade only (BD_CAC), under various offered load conditions. Fig. 6 to Fig. $\overline{9}$ are the blocking and dropping probabilities for UGS, ertPS, rtPS, and nrtPS connections. The results shows that the probability of dropping for hand over connections is always lower than that of blocking for new connections for all service types. This is what we desired in the design. Also, applying bandwidth degrade plus upgrade (the legend marked with BDR) does not cause ping-pong effect that makes the values changing drastically. Fig. 10 presents the utilization, the proposed BDR_CAC raises it to $82 \%$, comparing to $68 \%$ for CS_CAC and $65 \%$ for BD_CAC.

\section{CONCLUSIONS}

In this study, we utilized the concept of bandwidth quantization to design a BDR_CAC and reach the feasibility of analytical evaluation. The work can be further extended by taking delay constrain into account; that is, to design a CAC mechanism with both bandwidth and delay guarantee.

\section{REFERENCES}

[1] IEEE Std 802.16-2004, IEEE Standard for Local and Metropolitan Area Networks Part 16: Air Interface for Fixed Broadband Wireless Access Systems, October, 2004.

[2] IEEE Std 802.16-2009, IEEE Standard for Local and Metropolitan Area Networks Part 16: Air Interface for Broadband Wireless Access Systems, May, 2009.
[3] K. Wongthavarawat and A. Ganz, "Packet scheduling for qos support in IEEE 802.16 broadband wireless access systems," International Journal of Communication Systems, vol. 16, no. 1, pp. 81-96, 2003.

[4] H. Wang, B. He, and D. P. Agrawal, "Above packet level admission control and bandwidth allocation for IEEE 802.16 wireless man," Simulation Modelling Practice and Theory, vol. 15, no. 4, pp. 366-382, 2007.

[5] S. Andreev, Z. Saffer, A. Turlikov, and A. Vinel, "Upper bound on overall delay in wireless broadband networks with non real-time traffic," in Analytical and Stochastic Modeling Techniques and Applications, ser. Lecture Notes in Computer Science, K. Al-Begain, D. Fiems, and W. Knottenbelt, Eds. Springer Berlin / Heidelberg, 2010, vol. 6148, pp. 262-276.

[6] J. Chen, W. Jiao, and Q. Guo, "An Integrated Qos Control Architecture for IEEE 802.16 Broadband Wireless Access Systems," in Proc. IEEE Global Telecommunications Conference 2005, vol. 6, 2005, pp. 3330-3335.

[7] T.-C. Tsai, C.-H. Jiang, and C.-Y. Wang, "Cac and Packet Scheduling Using Token Bucket for IEEE 802.16 networks," Journal of Communications, vol. 1, no. 2, pp. 30-37, 2006.

[8] H. Wang, W. Li, and D. P. Agrawal, "Dynamic admission control and QoS for 802.16 wireless MAN," in Proc. Wireless Telecommunications Symposium, pp. 60 - 66, Apr. 2005.

[9] K. Wongthavarawat and A. Ganz, "Packet Scheduling for QoS Support in IEEE 802.16 Broadband Wireless Access Systems," in Proc. Military Communications Conference, IEEE 2003.

[10] S. Kalikivayi, I. S. Misra, and K. Saha, "Bandwidth and delay guaranteed call admission control scheme for QOS provisioning in IEEE 802.16e mobile WiMAX," in Proc. IEEE Conference on Global Telecommunications, 2008, pp. 1-6.

[11] C. T. Lea and A. Alyatama, "Bandwidth quantization and states reduction in the broadband ISDN," IEEE/ACM Trans. on Networking, vol. 3, no. 3, pp. 351-360, June, 1995.

[12] C. T. Lea and K. W. Ke, "Quantization, state reduction and cost-based admission and routing," Computer Communications, vol. 19, pp.1051-1064, 1996.

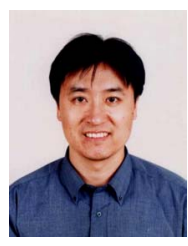

Kai-Wei Ke received the M.S. and Ph.D. degrees in electrical and computer engineering from Georgia Institute of Technology, USA, in 1996. Currently, he is a professor in the department of Computer Science and Information Engineering, National Taipei University of Technology, Taiwan, ROC. His research interests include traffic modeling, admission control, resource allocation, routing algorithms, and performance evaluation and applications for wireless multimedia communication networks.

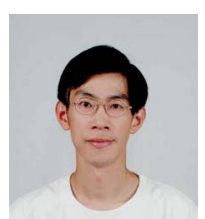

Ho-Ting Wu received his B.S. degree from National Taiwan University in 1986, and both the M.S. and Ph.D. degrees from University of California, Los Angeles in 1989 and 1994, all in electrical engineering. From 1986 to 1988 , he was an instructor at Naval Communication and Electronics School, Taiwan. From 1994 to 1996, he was a lecturer in the department of Electrical Engineering at Chang Gung College of Medicine and Technology, Taiwan. Since 1996, he has been on the faculty at National Taipei University of Technology, Taiwan, where he is currently a professor in the department of computer science and information engineering. His current research interests include multimedia communications, optical and wireless networks.

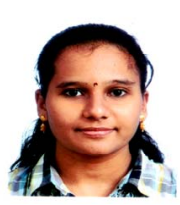

D. Jayasakthi is currently a Master student in the Department of Computer Science and Information Engineering, National Taipei University of Technology, Taiwan, ROC. Her research interests include computer networks and mobile applications.

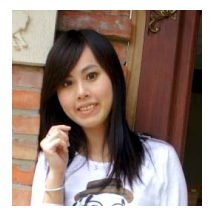

Shiao-Ting Huang received the Master degree from the Department of Computer Science and Information Engineering, National Taipei University of Technology, Taiwan, ROC. Currently, she is a software Engineer with Qisda Co. Ltd., Taiwan, ROC. 